http://dx.doi.org/10.4314/ajtcam.v10i1.22

\title{
ACARBOSE: A NEW OPTION IN THE TREATMENT OF ULCERATIVE COLITIS BY INCREASING HYDROGEN PRODUCTION
}

\section{De-Qing Zhang ${ }^{\&}$, Jian-Hong Zhu ${ }^{\&}$, Wei-Chang Chen*}

Department of Digestion Internal Medicine, the First Affiliated Hospital ,Soochow University,Su Zhou 215006, P.R. China.

*E-mail:chenweichang2012@163.com

\begin{abstract}
Acarbose, which is clinically widely used to treat Type 2 Diabetes, is thought to act at the small intestine by competitively inhibiting enzymes that delay the release of glucose from complex carbohydrates, thereby specifically reducing post prandial glucose excursion. The major side-effect of treatment with acarbose, flatulence, occurs when undigested carbohydrates are fermented by colonic bacteria, resulting in considerable amount of hydrogen. We propose that enteric benefits of acarbose is partly attributable to be their ability to neutralise oxidative stress via increased production of $\mathrm{H}_{2}$ in the gastrointestinal tract. Therefore, symptoms of ulcerative colitis in human beings can be ameliorated by acarbose.
\end{abstract}

Key words: acarbose, ulcerative colitis, hydrogen

\section{Introduction}

Ulcerative colitis (UC), with its causes still not fully understood, is a chronic inflammatory disease affecting the rectum and the colon to a variable extent and exerts a substantially negative impact on the quality of life of affected patients who suffer a $10 \%$ increased risk in developing colon carcinoma. Although some progress has been made in the treatment of UC in the last decades and many conventional medical treatments such as sulfaslazine,corticosteroids and immunosuppression are introduced into UC treatments from different aspects of UC etiologic process (Hawthorne et al.,2008; Carty,et al.,2003). Unfortunately, all these medicines have side-effects. People have to suffer from the sever adverse effects and the course of UC is still characterized by periods of remission interspersed with exacerbations (Solberg et al.,2008).

The expression of UC is generally believed to associate with the interplay of environmental, genetic and immunological factors (Fries et al.,2011). However, there is still not an integrated concept which explains the initiating event(s) and/or fundamental abnormalities in UC related to the pathophysiological changes. It does seem certain that UC is amplified and propagated by an uncontrolled and sustained host immune response, as the disease is paralleled by an extensive inflammatory infiltrate in the lamina propria, consisting of polymer phonuclear neutrophils, eosinophils and plasma cells. Whereas, the final steps resulting from such an excessive and enduring mucosal immune activation to tissue injury are still not fully confirmed. Ultimately, only a limited number of effector mechanisms, including reactive oxygen metabolites (ROM), might be responsible for the excessive cellular/tissue damage, chronic inflammation and destruction of normal tissue is observed in UC (Karp et al.,2006). Impaired antioxidant mechanism is implicated as one of pathogenic causes of UC. Inflamed tissues generate hydroxyl radicals, the most cytotoxic reactive oxygen species (ROS), which up-regulate TNF- $a$ expression through NF-kB signaling pathway and activate NADPH-Oxygenase (NOX) expression, increasing ROS production as a vicious circle (Gloire et al.,2006; Moe,et al.,2006).

In principle, excessive generation of ROM may lead to the attack and damage of all cellular and extracellular components (Roessner et al.,2008). However, it is important to realize that the overall high reactivity and short half-life of ROM implies that the inflicted tissue damage is generally close to the site of ROM generation. In UC, activated macrophages 
http://dx.doi.org/10.4314/ajtcam.v10i1.22

and neutrophils which produce excessive ROS aggregate in the inflaming gut and severe oxidative stress will occur subsequently. When exceeding the antioxidative ability of the intestinal antioxidant defense system, ROS will cause substantial damages in membrane lipid ,protein, and DNA, making the oxidative stress injuries in patients with UC worse and worse (Grisham et al.,1998). Since both inflammation and oxidation processes are reciprocally connected, antioxidants are viewed as a promising therapy for UC that can significantly alleviate the symptoms, i.e. body weight loss, visible fecal blood and diarrhea (HS et al.,2005). Therefore, the world has focused on finding new antioxidants in UC treatment .

\section{Molecular hydrogen $\left(\mathrm{H}_{2}\right)$ : a novel hotspot of antioxidant and inflammation suppressor}

In recent years, experimental evidences have proved that molecular hydrogen $\left(\mathrm{H}_{2}\right)$ acts as a novel antioxidant and inflammation suppressor with the following interesting properties(Ohsawa et al.,2007): (1) $\mathrm{H}_{2}$ can permeates cell membranes and target the cellular organelles, including the mitochondria and nuclei ( $\mathrm{Li}$ et al.,2012); (2) $\mathrm{H}_{2}$ specifically quenches detrimental ROS, such as ${ }^{\circ} \mathrm{OH}$ and peroxynitrite (ONOO-), while maintaining the metabolic oxidation-reduction reaction and their less-potent ROS, such as $\mathrm{O}_{2}^{-}, \mathrm{H}_{2} \mathrm{O}_{2}$ and nitric oxide (NO ) (Chen et al., 2011). It could be very important in clinics in the future that $\mathrm{H}_{2}$ could selectively react with exclusively detrimental ROS, such as hydroxyl radical and peroxynitrite, exerting protective effects. $\mathrm{H}_{2}$ did not react with other physiological ROS, such as superoxide anion and $\mathrm{H}_{2} \mathrm{O}_{2}$, which possess physiological roles. Therefore, unlike the other antioxidant supplements with strong reductive reactivity, $\mathrm{H}_{2}$ is mild enough without disturbing metabolic oxidation-reduction reactions or disrupting Oxidative Stress (OS) involved in cell signaling (Yoshida et al.,2012). Additionally, as $\mathrm{H}_{2}$ is electrically neutral and much smaller than the oxygen molecule, it easily penetrates membranes and enters cells and organelles such as nucleus and mitochondria (Kawai et al.,2012). This is particularly important, as the latter is the primary site of ROS generation and notoriously difficult to target, and hydrogen could diffuse freely within the body, without any side effects (Ohta.,2011). $\mathrm{H}_{2}$ can be administered orally in the form of $\mathrm{H}_{2}$-dissolved saline. Mikihito Kajiya reported that the administration of saturated hydrogen water for 7 days reduced the levels of several biomarkers of proinflammatory cytokine, such as IL-12 ,TNF- $\alpha$ and IL-1 $\beta$ (Kajiya et al., 2009). In other experiments, hydrogen gas treatment has significant protective effects on schistosomiasis associated chronic liver inflammation and $\mathrm{H}_{2}$-rich saline treatment significantly attenuates the severity of L-Arg-induced acute pancreatitis by ameliorating the increased serum amylase activity, inhibiting neutrophils infiltration and lipid oxidation (Chen et al.,2010). Therapeutic effects of $\mathrm{H}_{2}$ have been confirmed in the cell damage after stroke, ischemia-reperfusion injuries, transplantation injuries and other injuries related to oxidative stress (Hayashida et al.,2008; Fukuda,et al.,2007). Thus, $\mathrm{H}_{2}$ can protect cells from oxidative stress injuries. In inflammation process, $\mathrm{H}_{2}$ mediates suppression of proinflammatory cytolcines, especially IL-1 $\beta$, TNF- $\alpha$, IL-6 in inflammatory tissues. These research results suggest that persistent intake of $\mathrm{H}_{2}$ has the potential to reduce oxidative stress and may treat $\mathrm{UC}$.

\section{Acarbose : facilitate $\mathrm{H}_{2}$ generated in gastrointestinal tract and ameliorates symptoms of UC}

Acarbose is an $\alpha$-glucosidase inhibitor acting specifically at the level of postprandial glucose excursion. This compound lowers $\mathrm{HbA}_{1 \mathrm{c}}$ by $0.5-1 \%$ in patients with Type 2 diabetes, either drug naive or in combination with other antidiabetic drugs. In those with impaired glucose tolerance (IGT), it reduces the incidence of newly diagnosed diabetes by 36.4\% (Chiasson et al., 2003). Furthermore, it has beneficial effects on overweight, reduces blood pressure and triglycerides, and downregulates biomarkers of low-grade inflammation (Hanefeld et al.,2004). The major side-effect of treatment with $\alpha$-glucosidase inhibitors, flatulence, occurs when undigested carbohydrates are fermented by colonic bacteria, resulting in gas formation. The reason of flatulence is thought to act at the small intestine by competitively inhibiting enzymes that delay the release of glucose from complex carbohydrates, thereby specifically reducing post prandial glucose excursion. Then spontaneous production of $\mathrm{H}_{2}$ gas in the human body occurs via the fermentation of undigested carbohydrates by the resident enterobacterial flora (Yoshihiko et al., 2009). 
http://dx.doi.org/10.4314/aitcam.v10i1.22

$\mathrm{H}_{2}$ is not produced endogenously in mammalian cells, since the hydrogenase activity responsible for the formation of $\mathrm{H}_{2}$ gas may not be present (Stephen et al., 2012). Instead, spontaneous production of $\mathrm{H}_{2}$ gas in the human body occurs via the fermentation of undigested carbohydrates by the resident enterobacterial flora. $\mathrm{H}_{2}$ is transferred to the portal circulation and excreted through the breath in significant amounts (Michael et al.,1969). Flatulence is regarded as the major side-effect of treatment with acarbose (Ladas et al., 1992). Yoshihiko Suzuki reported that acarbose treatment significantly increased the amount of exhaled $\mathrm{H}_{2}$ by detecting in eleven healthy volunteers ( 10 males and 1 female) who were administered acarbose at a dosage of $300 \mathrm{mg} /$ day ( $100 \mathrm{mg}$ three times a day) for 4 days under free-feeding conditions. Kajiyama treated patients with type 2 diabetes or impaired glucose tolerance with $900 \mathrm{ml} /$ day $\left(300 \mathrm{ml}\right.$ three times a day) $\mathrm{H}_{2}$-dissolved water. After drinking $300 \mathrm{ml}$ of $\mathrm{H}_{2}$-dissolved water, the exhaled $\mathrm{H}_{2}$ gas concentration reached a maximum of $56 \pm 27.8 \mathrm{ppm}$ at 15 min, and returned to the baseline level at $150 \mathrm{~min}$ (Kajiyama et al.,2008). This peals level of $\mathrm{H}_{2}$ gas reduced the levels of oxidative stress biomarkers and improved glucose metabolism in patients with type 2 diabetes or impaired glucose tolerance. Considering that gastrointestinal tract derived $\mathrm{H}_{2}$, which is closely related to reduced cardiovascular events, could reduce general oxidative stress injuries, we assume that the induced $\mathrm{H}_{2}$ by acarbose might be the key to the symptoms alleviation in UC by cutting Myeloperoxidase (MPO) level.

According to the published data in 2009, mice UC induced by dextran sodium sulfate (DSS) was treated with saturated hydrogen water for 7 days. The study demonstrated that $\mathrm{H}_{2}$ could attenuate DSS-induced colitis by down-regulating the expression of proinflammatory cytokines, as well as suppressing the infiltration of macrophages in the colon lesion. The administration of $\mathrm{H}_{2}$ remarkably reduced the clinical symptoms of DSS-induced colitis, i.e., body weight loss, visible fecal blood and diarrhea, colitis score, and shortening of colon length. Histopathological evaluation further supported the effects of $\mathrm{H}_{2}$ on the prevention of DSS-mediated destruction of epithelium crypt structure. These observations clearly indicate that the amounts of $\mathrm{H}_{2}$ gas generated by acarbose are sufficient to reduce systemic oxidative stress. Oral administration of acarbose may be superior to drinking $\mathrm{H}_{2}$-rich water in terms of maintenance of the appropriate $\mathrm{H}_{2}$ gas levels in the body.

\section{Conclusion}

Based on these observations and experimental results, $\mathrm{H}_{2}$ has been proved effective in DSS-induced mice colitis and acarbose was thought to generate $\mathrm{H}_{2}$ in gastrointestinal tract .Therefore, we hypothesize that acarbose may be a novel and promising therapeutic option for UC as an indirect antioxidant. It may significantly restrict inflammation and alleviate clinical UC symptoms, improving the life quality of patients, and that these benefits can be attributed at least in part to the abilities of acarbose to neutralise oxidative stress by increasing the production of $\mathrm{H}_{2}$ in the gastrointestinal tract. Although acarbose probably has many other beneficial antioxidant effects on other diseases such as transplantation induced organs injury, cardiovascular diseases, cerebrovascular accidents etc., we still need to further study the biological mechanism.

\section{References}

1. B. Hawthorne, G Rubin, S. Ghosh.(2008).Review article: medication non-adherence in ulcerative colitis - strategies to improve adherence with mesalazine and other maintenance therapies. Alimentary Pharmacology \& Therapeutics.27:1157-1166.

2. Carty E, Rampton DS. (2003).Evaluation of new therapies for inflammatory bowel disease. J Clin Pharmacol .56:351-361.

3. IC Solberg, I Lygren, J Jahnsen. (2008) .Clinical course during the first 10 years of ulcerative colitis: results from a population-based inception cohort (IBSEN Study). Scandinavian Journal of Gastroenterology.27:1157-1166.

4. Fries, Walter, Comunale,et al. (2011).Ulcerative Colitis:Pathogenesis.Current Drug Targets. 12:1373-1382.

5. Karp SM, Koch TR. (2006). Oxidative stress and antioxidants in inflammatory bowel disease. Dis Mon.52:199-207. 
http://dx.doi.org/10.4314/ajtcam.v10i1.22

6. Gloire G, Legrand-Poels S, Piette J. (2006). NF-kappaB activation by reactive oxygen species: fifteen years later. Biochem Pharmacol.72:1493-1505.

7. Moe KT, Aulia S, Jiang F, et al. (2006). Differential upregulation of Nox homologues of NADPH oxidase by tumor necrosis factoralpha in human aortic smooth muscle and embryonic kidney cells. J Cell Mol Med.10:231-239.

8. A Roessner, D Kuester, P Malfertheiner. (2008).Oxidative stress in ulcerative colitis-associated carcinogenesis. Research and Practice.204:511-524.

9. Grisham MB, Granger DN. (1998).Neutrophil-mediated mucosal injury: role of reactive oxygen metabolites. Dig Dis Sci.33:6S-15S.

10. Oz HS, Chen TS, McClain CJ, et al. (2005).Antioxidants as novel therapy in a murine model of colitis. J Nutr Biochem.16:297-304.

11. Ohsawa I, Ishikawa M, Takahashi K, et al. (2007).Hydrogen acts as a therapeutic antioxidant by selectively reducing cytotoxic oxygen radicals. Nat Med.13: 688-694.

12. G Li, M Ji, X Sun. (2012) .Effects of hydrogen-rich saline treatment on polymicrobial sepsis. Journal of Surgical Research .http://dx.doi.org/10.1016/j.jss.2012.06.058.

13. Chen XA, Zuo QA, Hai YD, et al. (2011). Lactulose: An indirect antioxidant ameliorating inflammatory bowel disease by increasing hydrogen production. Medical hypotheses. 76:325-332.

14. Yoshida A, Asanuma H, Sasaki H. (2012).H2 Mediates Cardioprotection Via Involvements of KATP Channels and Permeability Transition Pores of Mitochondria in Dogs. Cardiovasc Drugs Ther. 26:217-226.

15. Kawai D, Takaki A, Nakatsuka A. (2012).Hydrogen-rich water prevents progression of non-alcoholic steatohepatitis and accompanying hepatocarcinogenesis in mice. Hepatology.doi: 10.1002/hep.25782.

16. S Ohta. (2011).Molecular hydrogen is a novel antioxidant to efficiently reduce oxidative stress with potential for the improvement of mitochondrial diseases. Biochimica et Biophysica Acta. doi:10.1016/j.physletb.2003.10.071

17. Kajiya M, Silva MJ, Sato K, et al. (2009). Hydrogen mediates suppression of colon inflammation induced by dextran sodium sulfate. Biochem Biophys Res Commun .386:11-15.

18. Chen H, Sun YP, Li Y, et al. (2010).Hydrogen-rich saline ameliorates the severity of L-arginine-induced acute pancreatitis in rats. Biochem Biophys Res Commun.. 393:308-313.

19. Hayashida K, Sano M, Ohsawa I, et al. (2008).Inhalation of hydrogen gas reduces infarct size in the rat model of myocardial ischemia-reperfusion injury. Biochem Biophys Res Commun. 373:30-35.

20. Fukuda K, Asoh S, Ishikawa M, et al. (2007).Inhalation of hydrogen gas suppresses hepatic injury caused by ischemia/reperfusion through reducing oxidative stress. Biochem Biophys Res Commun.361:670-674.

21. Chiasson J L,Josse, R G., Gomis R., et al. (2003). Acarbose treatment and the risk of cardiovascular disease and hypertension in patients with impaired glucose tolerance: the STOP-NIDDM trial. JAMA.290: 486-494.

22. Hanefeld M., Cagatay M., Petrowitsch T. (2004).Acarbose reduces the risk for myocardial infarction in type 2 diabetic patients: meta-analysis of seven long-term studies. European Heart Journal.25: 10-16.

23. Yoshihiko Suzuki,Motoaki Sano c, Kentaro Hayashida,et al. (2009). Are the effects of a-glucosidase inhibitors on cardiovascular events related to elevated levels of hydrogen gas in the gastrointestinal tract? FEBS Letters.83:2157-2159.

24. Stephen A. McClave. (2012). Drivers of Oxidative Stress in Acute Pancreatitis. Journal of Parenteral and Enteral Nutrition. 36: 24-35.

25. Michael D, Levitt, M D. (1969).Production and excretion of hydrogen gas in man. New England Journal of Medicine.281:122-127.

26. S D Ladas, A Frydas, A Papadopoulos, et al. (1992). Effects of $\alpha$-glucosidase inhibitors on mouth to caecum transit time in humans. Gut..33:1246-1248.

27. S Kajiyama,G Hasegawa, M Asano,et al. (2008). Supplementation of hydrogen-rich water improves lipid and glucose metabolism in patients with type 2 diabetes or impaired glucose tolerance. Nutr. Res. 28: 137-143. 